\title{
Représentation de la féminité à travers les adaptations a l'écran du roman Le mariage de Chiffon
}

\author{
Ángela Magdalena Romera Pintor \\ Universidad Nacional de Educación a Distancia \\ aromera@flog.uned.es
}

Rebut: 16 desembre de 2014

Acceptat: 2 d'abril de 2015

RESUM

Representació de la feminitat a través de les adaptacions a la pantalla de la novel-la Le mariage de Chiffon

Aquest article tracta dels personatges femenins d'una novel-la de Gyp, Le mariage de Chiffon, a través de l'anàlisi de la seva representació en dues adaptacions per a la petita i la gran pantalla. D'aquesta manera es pot establir una imatge diferent de la feminitat en tres moments separats successivament en el temps per un interval d'un mig segle: el de la novel-la, de 1894; el de la pel-lícula en blanc i negre de 1942, amb Odette Joyeux en el paper de Chiffon i Suzanne Dantès en el de Madame de Bray; i el del telefilm de France Télévision, de 2010, que se emmarca en la sèrie Au siècle de Maupassant, Contes et Nouvelles du XIX ${ }^{e}$ siècle, amb Christa Théret en el paper de Chiffon i Christiane Millet en el de Madame de Bray. L'estudi permetrà observar l'evolució de la representació de la feminitat, del text escrit a la pantalla, des del final del segle XIX fins a principis del segle XXI.

Mots CLAU

Escriptura femenina francesa, novel-la sentimental, Gyp, representació femenina, adaptacions a la pantalla.

RÉSUMÉ

Représentation de la féminité à travers les adaptations à l'écran du roman Le mariage de Chiffon

Cet article étudie les personnages féminins d'un roman de Gyp, Le mariage de Chiffon, à travers l'analyse de leur représentation dans deux adaptations à l'écran. Nous serons à même d'établir une image différente de la féminité à 
trois moments séparés successivement dans le temps par un écart d'environ un demi siècle : celui du roman, de 1894 ; celui du film en noir et blanc de 1942 , avec Odette Joyeux dans le rôle de Chiffon et Suzanne Dantès dans celui de Madame de Bray ; et celui du téléfilm de France Télévision, de 2010, qui s'intègre dans la série Au siècle de Maupassant, Contes et Nouvelles du XIX siècle, avec Christa Théret dans le rôle de Chiffon et Christiane Millet dans celui de Madame de Bray. Cette analyse nous permettra de retracer l'évolution de la représentation de la féminité, du texte écrit à l'écran, de la fin du XIX ${ }^{\mathrm{e}}$ siècle au début du XXI ${ }^{e}$ siècle.

\section{MoTS CLÉ}

Écriture féminine française, roman sentimental, Gyp, représentation féminine, adaptations à l'écran.

\section{RESUMEN}

Representación de la feminidad a través de las adaptaciones para la pantalla de la novela Le mariage de Chiffon

Este artículo aborda el estudio de los personajes femeninos de una novela de Gyp, Le mariage de Chiffon, a través del análisis de su representación en dos adaptaciones para la pequeña y la gran pantalla. De esta manera, podremos establecer una imagen diferente de la feminidad en tres momentos separados sucesivamente en el tiempo por un intervalo de aproximadamente medio siglo: el de la novela, de 1894; el de la película en blanco y negro de 1942, con Odette Joyeux en el papel de Chiffon y Suzanne Dantès en el de Madame de Bray; y el del telefilm de France Télévision, de 2010, que se enmarca en la serie Au siècle de Maupassant, Contes et Nouvelles du XIX ${ }^{e}$ siècle, con Christa Théret en el papel de Chiffon y Christiane Millet en el de Madame de Bray. Este análisis nos permitirá desentrañar la evolución de la representación de la feminidad, del texto escrito a la pantalla, desde el final del siglo XIX hasta principios del siglo XXI.

\section{PALABRAs Clave}

Escritura femenina francesa, novela sentimental, Gyp, representación femenina, adaptaciones a la pantalla.

\section{Abstract}

Representation of femininity in the adaptations for the screen of the novel

\section{Le mariage de Chiffon}

This article studies the female characters of Gyp's novel, Le mariage de Chiffon, and how they are represented in two adaptations for the screen. Our exploration 
will enable us to ascertain the portrayed images of femininity in three distinct moments of time, each one more or less separated by fifty years: that of the novel, in 1894; that of the film in black and white of 1942 (with Odette Joyeux in the role of Chiffon and Suzanne Dantès in the role of Madame de Bray); and finally that of the TV adaptation of France Télévision, in 2010, which forms part of the series Au siècle de Maupassant, Contes et Nouvelles du XIXe siècle (with Christa Théret in the role of Chiffon and Christiane Millet in the role of Madame de Bray). This analysis will allow us to unveil the evolution of feminine representation, from the written text to the screen, from the end of the $19^{\text {th }}$ century and through to the beginning of the $21^{\text {st }}$ century.

\section{KEYWORDS}

French feminine writing, sentimental novel, Gyp, feminine representation, screen adaptations.

\section{Le roman de 1894: Le mariage de Chiffon}

Gyp, n'a pas besoin de présentation. Cependant, la célébrité que cette femme de lettres obtint dans son temps, non seulement pour ses œuvres offrant un tableau satirique des mœurs et de la société, mais aussi pour ses caricatures sociales $^{1}$ et sa participation dans les débats politiques et publiques, serait bientôt effacée au profit surtout d'un seul ouvrage, Le mariage de Chiffon, de 1894, qui a été invariablement identifié avec le roman sentimental'2. Il s'agit, en fait, du seul des romans de la comtesse de Martel qui éveille encore de

\footnotetext{
${ }^{1}$ Après la mort de Gyp (1849-1932), son ami Lucien Corpechot lui dédie un article publié dans la Revue de Paris, où il souligne justement cet aspect : « Gyp, comme tous les grands observateurs de notre race, se servait pour regarder le monde de ce merveilleux instrument d'optique qu' on pourrait appeler le télescope Molière : il grossit sans déformer. C'est la marque de nos grands caricaturistes [...]. Gyp excellait dans la caricature. Paris s'est longtemps délecté aux dessins de Bob, pleins d'esprit, de malice, de courage, et d'un sens si parfaitement exact » (Lucien CORPECHOT, «Le souvenir de Gyp », La revue de Paris, Trente-neuvième année, Tome quatrième, juillet-août 1932, p. 438).

${ }^{2}$ Les ouvrages de Gyp considérés « sentimentaux » sont ceux qui seront associés à la survie de sa célébrité. Cf. dans ce contexte, le Dictionnaire des femmes célèbres : « Mais c'est avec le genre sentimental qu'elle devait passer à la postérité ; en effet, Le Mariage de chiffon (1894) remporta un considérable succès et fut porté à l'écran » (Lucienne MAZENOD, et Ghislaine SCHOELLER, Dictionnaire des femmes célèbres, Éditions Robert Laffont, Paris, 1992, p. 381).
} 
nos jours un certain intérêt populaire, comme le prouvent ses rééditions et ses adaptations à l'écran ${ }^{3}$, les plus représentatives étant celles du film de 1942 et de la série télévisée de 2010, dont nous nous occuperons plus tard.

Toutefois, s'il est vrai que Le mariage de Chiffon continue à être identifié avec le roman sentimental à cause du sujet traité et du dénouement heureux de l'histoire (l'amour d'une jeune fille qui finit par épouser l'homme qu'elle aime, malgré la différence d'âge), il faut préciser aussi qu'il s'écarte considérablement de ce genre populaire en raison du traitement que l'auteure accorde aux discours et aux propos qu'il contient. De ce fait, le roman partage de nombreux traits avec l'écriture incisive de l'ensemble de l'œuvre de Gyp, qui regorge de verve satirique et dont l'esprit contestataire découle d'une observation critique des mœurs, de la société et du milieu dont elle-même faisait partie.

D'emblée, il convient de signaler que l'écrivaine fait dérouler l'histoire de Chiffon dans une ville de province romancée, Pont-sur-Sarthe, qui lui fournira l'occasion de dénoncer la bourgeoisie et la petite aristocratie provinciales, avec leurs ambitions, affectations et mesquineries. La romancière y manifestera aussi son admiration avouée pour Napoléon, dont la défense sera prise en charge par l'héroïne du roman, ainsi que son antipathie à l'égard des protestants et des Jésuites, ces derniers étant représentés ici par le père de Ragon, personnage qui sera impitoyablement fustigé à cause de son penchant pour l'argent, les relations sociales et les intrigues. Il est à noter, en tout cas, que le discours critique de cet ouvrage nous est surtout transmis au moyen des dialogues qui occupent la presque totalité du texte.

\footnotetext{
${ }^{3}$ D'après l'information recueillie dans IMDb il y aurait aussi une version italienne, qui serait, en fait, la toute première version filmée de ce roman de Gyp puisqu'elle date de 1918. Elle fut dirigée par Alberto Carlo Lolli et interprétée par Mary Bayma-Riva dans le rôle de Chiffon.

Cf. http://www.imdb.com/title/tt0794312/fullcredits?ref_=tt_cl_sm\#cast.

Une autre adaptation dont on ne s'occupera point dans notre article mais qui prouve bien la popularité de ce roman de Gyp est celle qui fait partie de la série télévisée Les amours de la Belle Époque, série réalisée par René Lucot en 1979 et composée de 65 épisodes. L'épisode qui s'occupe du Mariage de Chiffon fut dirigé par Agnès Delarive, avec Magali Renoir dans le rôle de Chiffon, Francine Bergé dans le rôle de la marquise, et Maïa Simon dans celui de Mme de Liron. Cf. http:// www.ina.fr/video/CPB79056414, qui offre l'entretien avec Henri Spade, producteur et responsable pour Antenne 2 de ladite série et qui commente Les amours de la Belle Époque dans le programme Face à vous, émis en 1979. Il y explique pourquoi il a choisi des « romans sentimentaux » pour cette série, où chaque épisode n'est qu'une « séquence d'un quart d'heure qui passe tous les jours avant le journal de 13 heures ». De son côté, Robert Cuzin -qui représente les lecteurs de Télé 7 jours- intervient dans le programme pour y offrir les commentaires des spectateurs.
} 


\section{Les personnages féminins du roman}

Signalons tout d'abord que nous ne nous occuperons ici que des trois personnages féminins les plus représentatifs du roman : l'hérö̈ne, Chiffon ; la mère de celle-ci, Madame de Bray; et la rivale de Chiffon, Madame de Liron.

Il y a, nous l'avons déjà signalé, de rares intervalles dans Le mariage de Chiffon où le discours narratif prend le devant sur le dialogué et cela seulement pour offrir au lecteur les informations essentielles de l'histoire, telles que la description des personnages, l'explication de certains propos et les quelques incidents qui permettent d'avancer dans le déroulement de l'action. C'est ainsi que le roman débute avec un dialogue qui s'étale tout le long du premier chapitre et qui permet d'introduire non seulement le fond de l'intrigue, mais aussi une succession impressionniste de traits, éparpillés parmi les échanges dialogiques, nous découvrant petit à petit les personnages principaux de l'ouvrage.

La description du physique de l'hérö̈ne est étalée de la sorte dans ce premier dialogue par des petites touches significatives : Chiffon a des « yeux d'un gris ${ }^{4}$ très pâle $»^{5}$, « des cils bruns étonnamment longs et touffus $»^{6}$, un " petit visage chiffonné $»^{7}$, les cheveux très blonds, " Coryse secoua sa tête trop blonde $»^{8}$, et des $«$ joues fraîches $»^{9}$. L'âge de la fillette nous est révélé par elle-même, qui affirme dans une de ses interventions : « je n'ai que seize ans et demi $! »^{10}$. Ce ne sera qu'une fois le dialogue bien entamé que l'auteure fournira une description posée de Coryse, plus conforme au format habituel des romans sentimentaux :

Corysande D'Avesnes, qu'on appelait Coryse, ou plus habituellement Chiffon, était une fillette solide et souple, beaucoup plus bébé que jeune fille, avec encore les angles et les disproportions de l'enfance, et la peau transparente

\footnotetext{
${ }^{4}$ Les yeux de Chiffon nous seront dépeints un peu plus tard d'une couleur bleue : « Les doux yeux bleus se troublaient, et des larmes rondes, semblables à des boules de verre, glissaient sans déformer sur les joues fraîches de Coryse » (Gyp, Le Mariage de Chiffon, Nelson Éditeurs \& Calmann-Lévy Éditeurs, Paris, 1917, p. 13) ; «Chiffon le suivait de son regard bleu devenu tout pensif » (Ibid., p. 218).

${ }^{5}$ Ibid., p.8.

${ }^{6}$ Ibid., p. 8.

${ }^{7}$ Ibid., p. 9.

${ }^{8}$ Ibid., p. 10.

${ }^{9}$ Ibid., p. 13.

${ }^{10}$ Ibid., p. 13.
} 
des tout petits, - cette peau sous laquelle courent des lueurs roses. - Ses mouvements harmonieux et agiles, bien qu'un peu maladroits, qui rappelaient ceux d'un grand jeune chien, irritaient sa mère autant presque que son langage trop peu correct ${ }^{11}$.

Remarquons aussi que le détail des cheveux blonds et flottants de Coryse — « La petite secoua la tête, faisant voler se cheveux légers $»^{12}$ —, souvent ébouriffés — « en secouant avec découragement sa tête ébouriffée ${ }^{13}$ —, ou tout simplement couverts d'un chapeau ou d'une capeline de paille au moment de sortir — « Chiffon avait bondi dans sa chambre, planté de travers un chapeau sur sa toison blonde ${ }^{14}$ — devient de tous les traits qui la caractérisent celui qui l'identifie le mieux et celui qui lui sera affiché de façon systématique tout le long du roman : « en voyant les cheveux flottants et la petite frimousse aimée qui lui souriait, il se rendit compte que c'était bien "le Chiffon" qui était devant lui ${ }^{15}$. Les cheveux de l'héroïne font partie, en outre, d'un de ses attraits féminins les plus remarquables : « La nappe de cheveux blonds qui flottait autour d'elle, envolée au courant d'air de la fenêtre, lui donnait l'aspect d'une petite fée, d'un petit être bizarre et irréel ${ }^{16}$. Mais ils sont aussi et surtout un motif récurrent qui permet de symboliser son jeune âge, sa proximité avec l'enfance même. Cette image de Coryse, avec ses longs cheveux blonds en liberté, s'avère ainsi la représentation que l'auteure développe par excellence pour mettre en exergue la fraîcheur naturelle, l'extrême jeunesse — voire enfance $^{17}$ — et la spontanéité de son héroïne, qui est au demeurant une « gamine à la fois svelte et râblée, rêveuse et gavroche ${ }^{18}$ :

Le duc d'Aubières, lui, était resté un peu ému et décontenancé. Il s'attendait si peu à trouver là Chiffon, _ qui jamais n'allait nulle part, — et il s'attendait si

\footnotetext{
${ }^{11}$ Ibid., p. 13-4.

${ }^{12}$ Ibid., p. 240.

${ }^{13}$ Ibid., p. 17.

${ }^{14}$ Ibid., p. 29.

${ }^{15}$ Ibid., p. 243.

${ }^{16}$ Ibid., p. 285.

${ }^{17}$ En fait, Gyp prend plaisir à comparer son héroïne même avec un « bébé », notamment quand elle se cramponne au cou de son oncle Marc ou qu'elle lui grimpe sur les genoux : « mais laisse-moi donc tranquille, grande bête !... — ajouta-t-il en se levant brusquement, faisant glisser Coryse, qui lui grimpait sur les genoux comme un bébé » (Ibid., p. 21).
}

${ }^{18}$ Ibid., p. 54. 
peu surtout à la voir presque femme, bien habillée, ne gardant de l'enfant que les longs cheveux flottants sur les épaules ${ }^{19}$.

Il existe un dernier trait physique qui singularise Coryse et qui pourrait surprendre à un lecteur assidu aux romans d'amour, celui de sa myopie, imperfection q-ue le lecteur apprendra au troisième chapitre : « Coryse s'arrêta, examinant, dans le clignement familier aux myopes, les gens qui causaient $»^{20}$. Ce défaut physique sera repris plusieurs fois dans le texte comme pour indiquer qu'il fait partie de la nature du personnage et qu'il ne s'agit point d'une étourderie que l'auteure aurait glissé dans son récit par mégarde. Il s'agit, en conséquence, d'un détail qui s'avère d'autant plus important qu'il relie le personnage avec la réalité, toujours imparfaite, face à la perfection physique des jeunes protagonistes féminines de la vaste majorité des romans d'amour, comme ceux de Delly, qui n'admet aucune imperfection corporelle chez ses hérö̈nes.

D'autre part, le langage colloquial de Chiffon se veut tout d'abord spontané, frais, naturel, et de ce fait, associé une fois de plus à l'innocence et à l'enfance. Il sera aussi, néanmoins, réactionnaire du moment qu'il s'avère une arme employée par Chiffon pour désarçonner les prétentions d'élégance et de savoir faire de sa mère, la marquise de Bray. Le discours de la fillette frappe à l'oreille et lui sera reproché maintes fois dans le roman au moyen d'autres personnages de son entourage, tels que sa mère ou sa tante. Toutefois, malgré son parler argotique, l'héroïne développe des discours raisonnés et réfléchis, bien que souvent trop sincères pour ne pas offenser la bienséance des mœurs. Ainsi, le langage de Coryse semble entretenir un rapport direct avec ses réactions et ses propos, toujours francs et souvent impulsifs. Il n'est donc point surprenant que ce soit la propre Coryse qui à la fin de l'histoire propose à Marc de l'épouser : «Ben, moi... je pourrais t'en indiquer une à faire... et pas loin... de belle action ?... Et, comme il ne répondait pas, elle murmura dans un faible souffle : - Ça serait de m'épouser ? $»^{21}$.

Tout compte fait, la petite Coryse partage de nombreux traits avec son auteure : une mère autoritaire et superficielle, un langage direct et contestataire, sans fade et dépourvu de duplicité, un esprit indépendant et critique, et un amour passionné pour les fleurs ${ }^{22}$ et les animaux, outre sa passion pour Napoléon

\footnotetext{
${ }^{19}$ Ibid., p. 244.

${ }^{20}$ Ibid., p. 51.

${ }^{21}$ GYP, op. cit., p. 285.

${ }^{22}$ L'amour de la comtesse de Martel pour les fleurs et les animaux est rappelée dans l'article que son ami Corpechot publia dans La revue de Paris: «Gyp qui était moqueuse et impitoyable pour
} 
déjà commentée, et son aversion ${ }^{23}$ à l'égard des Jésuites et des protestants, que Gyp avait manifestées aussi de son vivant dans d'autres écrits, comme ses mémoires.

De son côté madame de Bray est surtout dépeinte comme une femme superficielle et prétentieuse. Les traits physiques de ce personnage ne nous sont pas fournis, mais sa beauté est mentionnée au passage lors de l'explication du déroulement de ses secondes noces : «Elle commençait à mûrir et comprenait que sa beauté, toute de fraîcheur et d'éclat, allait disparaître tout à coup $»^{24}$. Toutefois, même si Gyp s'empresse de la présenter peu après comme étant « très vulgaire d'allure et d'aspect ${ }^{25}$, l'auteure fera allusion dans d'autres occasions à la beauté de la marquise : " toutes les plus jolies femmes - y compris madame de Bray à son déclin, mais encore appétissante (...) $\gg^{26}(54)$. Mais ce sera surtout à travers ses tenues, conçues pour impressionner, que son apparence nous sera le mieux représentée :

Totalement dénuée de goût ; incapable de discerner la grâce d'une robe bien coupée de la laideur d'une robe mal faite (...), la toilette féminine se réduisait pour elle « à ce qui fait de l'effet» ou « n'en fait pas ». (...) Donc madame de Bray achetait des étoffes et faisait faire, chez des ouvrières borgnes de Pont-surSarthe, des robes qui allaient épouvantablement. ${ }^{27}$

Si la description physique de madame de Bray est à peine effleurée dans le roman par ces quelques traits, son caractère, en revanche, nous y est dépeint avec insistance : « Entichée de noblesse, — et d'argent aussi depuis qu'elle en avait, — aimant pardessus tout le panache et la pose, elle ne pardonnait pas à la petite Coryse une simplicité et une rondeur qu'elle ne comprenait point $»^{28}$. Son regard, ses poses et le ton de ses interventions sont soigneusement décrits

les ridicules des hommes n'avait que de la tendresse pour les bêtes et elle ne pouvait voir souffrir une fleur » (Corpechot, op. cit., 437-8).

De son côte, l'amour de Chiffon pour les fleurs est relevé à plusieurs reprises dans le roman : «Car Chiffon, persuadée que les fleurs souffrent, ne les touchait qu' avec une délicatesse infinie et d'attendrissantes précautions » (Gyp, op. cit., 60).

${ }^{23}$ Toutefois, on ne retrouve pas dans cet ouvrage l'habituel discours contre les juifs, auxquels cependant l'écrivaine avait dédié des critiques acharnées.

${ }^{24}$ Ibid., p. 15.

${ }^{25}$ Ibid., p. 16.

${ }^{26}$ Ibid., p. 54.

${ }^{27}$ Ibid., p. 228-9.

${ }^{28}$ GYP, op.cit., p. 16. 
et qualifiés, la plupart du temps pour mettre en relief son animadversion envers Coryse et l'antagonisme qui les sépare. En fait, les frictions entre mère et fille dérivent de leurs caractères foncièrement antithétiques : la franchise naturelle et spontanée de Chiffon, son absence de prétention, de fourberie, sa bonté de cœur et sa simplicité contrastent vivement avec l'attitude et les allures hypocrites et affectées de madame de Bray, préoccupée surtout par le statut social, le «qu'en dira-t-on » et les apparences. Les mièvreries de la marquise deviennent ainsi la cible préférée des critiques de Gyp : «Et se levant, d'un mouvement qu'elle croyait très noble et qui était très ridicule, la marquise sortit à grands pas du salon $»^{29}$. Cette femme se montrera absolument insupportable, non seulement avec Coryse, mais aussi avec son deuxième mari, qui de ce fait établira des liens d'amitié avec sa belle-fille : « Tout de suite, M. de Bray aima Chiffon (...). L'intraitable caractère de sa femme amena ce rapprochement. Effarouchés du vacarme, des pleurs, des éclats et des grands gestes de la marquise, ces deux êtres gais et bons cherchèrent instinctivement l'un chez l'autre un appui $»^{30}$. Il est vrai, cependant, que vers la fin de l'ouvrage Gyp finira par faire des concessions à ce personnage, à l'égard duquel elle n'éprouve de toute évidence aucune sympathie : "Quant à la marquise, l'admiration inspirée par sa fille la ravit absolument. Pas du tout mauvaise au fond, mais seulement vaine et sotte, elle jouissait pleinement de tout ce qui contribuait en quelque sorte à la grandir et à la mettre en vue $»^{31}$. Cette caractérisation de la marquise ( « vaine et sotte », mais « pas du tout mauvaise au fond ») devient ainsi la moins critique du roman et peut-être aussi celle qui condense le mieux sa personnalité.

Quant à la rivale de l'héroïne, il est important de noter que, tout en partageant les principales caractéristiques de ce personnage prototypique des romans d'amour, elle ne jouera qu'un rôle très limité dans l'histoire. Pour commencer, c'est une femme mariée, ce qui réduit considérablement les possibilités effectives de concurrence avec Coryse, qui est célibataire. Une brève allusion au premier chapitre nous apprend son existence : «- $\mathrm{Si}$ j'en crois les potins... la petite de Liron t'adore... et elle a vingt ans de moins que toi ?... - En admettant que ce soit... elle m'adore aujourd'hui, mais demain ? $»^{32}$. Cette remarque nous découvre son rapport avec l'oncle $\operatorname{Marc}^{33}$,

\footnotetext{
${ }^{29}$ Ibid., p. 11.

${ }^{30}$ Ibid., p. 38-9.

${ }^{31}$ Ibid., p. 241.

${ }^{32}$ Ibid., p. 23.

${ }^{33}$ Même si Marc est présenté comme l'oncle de Coryse, il n'a aucun rapport de famille avec elle, autre que celui d'être le frère de son beau-père : « - Pourquoi as-tu été si méchant ?...
} 
le héros de l'histoire. Même Coryse a entendu parler de l'infidélité de cette dame grâce aux racontars habituels qui circulent à Pont-sur-Sarthe : «Encore une qui trompe son mari, madame de Liron $! »^{34}$. Cependant, ce personnage n'apparaîtra que vers la fin du roman, chez « la première couturière » de la ville, où Marc amène Chiffon pour lui offrir une robe : « la petite de Liron, enveloppée d'un nuage de gaze rose, entra en tourbillon $»^{35}$. Il est intéressant de constater que la description physique de cette femme - souvent qualifiée de «petite » à cause de sa taille menue — est l'une des plus développées de l'ouvrage :

C'était une très jolie petite personne rondelette et capitonnée de fossettes. Ses cheveux bruns frisottaient sur un front plat aux contours mous. Elle avait de grands yeux chocolat très câlins, un nez correct, une toute petite bouche, (...) et un teint superbe. Les épaules sortaient blanches et grasses de la robe décolletée à l'excès. Le haut des bras s'engorgeait un peu. L'oreille plate et incolore s'attachait mal, trop renversée et trop éloignée des cheveux.

Telle quelle, Chiffon comprenait, - bien qu'elle n'aimât pas ce genre de femme, - que madame de Liron était très jolie et devait plaire beaucoup ${ }^{36}$.

Elle apparaîtra encore brièvement à deux reprises : chez les Barfleur, et plus tard chez Coryse, lors du bal organisé par madame de Bray, dernier épisode du roman. Son attitude, sa conduite et ses allures dans ces deux occasions dévoilent son penchant pour les hommes et les flirts. On la retrouve ainsi occupant « le centre du groupe formé par les hommes » (248). Habituée à être le centre d'attentions, elle aime à être admirée : « Madame de Liron n'aimait à valser que pour les spectateurs $»^{37}$. Elle sait aussi tirer le meilleur parti de sa personne pour éblouir dans les événements sociaux, non seulement à travers sa toilette, mais aussi par sa conduite calculée : "Mais la petite de Liron désirait avant tout se faire voir au comte Axen "dans son beau jour", et elle savait que les petits hommes ne font pas valoir les femmes qui dansent avec

pourquoi m'as-tu dit que tu n'es pas mon oncle ?... - Mais parce que, bien que je t'aime autant que si je l'étais, je ne le suis pas !... je suis le frère du mari de ta mère... je ne te suis rien... je pourrais t'épouser... si je n'étais pas de l'âge de ton ami d'Aubières, que tu envoies si gentiment promener... » (Ibid., p. 24).

${ }^{34}$ Ibid., p. 231.

${ }^{35}$ Ibid., p. 232.

${ }^{36}$ GYP, op. cit., p. 233.

${ }^{37}$ Ibid., p. 255. 
eux $»^{38}$. Finalement, comme toutes les rivales d'usage des romans d'amour, Madame de Liron se montrera jalouse à l'égard de l'héroïne, qui vers la fin du roman s'est transformée de façon spectaculaire grâce à la nouvelle robe offerte par l'oncle Marc : «La jeune femme venait d'apercevoir Coryse. (...) Son joli visage riant prit une expression d'effarement mauvais $»^{39}$. L'intérêt que Madame de Liron éprouve pour Marc est à l'origine de l'antipathie réciproque que ces deux personnages féminins se manifestent. Ainsi, Coryse trouve la petite de Liron «trop rondouillarde ${ }^{40}$ et toujours déplaisante : "Rien dans cette rondelette poupée, aux yeux polissons, aux lignes un peu vulgaires, ne plaisait à Chiffon $\gg^{41}$. La jeune fille, sans savoir trop pourquoi, éprouvera un sentiment de rejet en sa présence: « depuis que je sais qu'elle y sera... ça me paraît encore plus bassin ! $\gg^{42}$. De ce fait, Chiffon s'opposera à elle de façon systématique : " Madame de Liron avait dit tout à l'heure à l'oncle Marc : "Vous allez lui faire quelque chose de rose, j'espère ?..." Cela seul suffisait pour déterminer la petite à choisir n'importe quelle couleur, excepté celle-là » ${ }^{43}$.

Cependant, tel que nous l'avons déjà signalé, la place que Gyp accorde à Madame de Liron dans le roman est minimale. Tout compte fait, la présence de cette rivale ne semble jouer qu'un seul rôle dans l'histoire : celui d'éveiller chez l'hérö̈ne des sentiments d'inquiétude qui la conduiront à comprendre qu'elle est amoureuse de son oncle Marc.

Voilà donc la représentation littéraire de ces trois figures féminines : Chiffon, jeune fille presque enfant, avec un esprit indépendant et rebelle, souvent contestataire et de ce fait effronté, mais simple, fraîche et spontanée, au cœur pur et bon. Elle s'avère en fait une représentation assez vraisemblable de l'adolescence, mais aussi et surtout de Gyp elle-même ; Madame de Bray, vaine et sotte, superficielle et affectée, égocentrique et ridicule dans ses grands airs de dame. Elle représente l'hypocrisie de la société mondaine; et Madame de Liron, jolie mais égoïste et vaine, avec le seul but de plaire aux hommes appartenant à un niveau social élevé. Elle évoque l'arrivisme et la vanité. Il s'agit en tout cas de personnages féminins qui permettent à l'auteur de mieux verser sa critique sociale au moyen de figures féminines présentes dans la réalité de son temps.

\footnotetext{
${ }^{38}$ Ibid., p. 272.

${ }^{39}$ Ibid., p. 235.

${ }^{40}$ Ibid., p. 256.

${ }^{41}$ Ibid., p. 273.

${ }^{42}$ Ibid., p. 235.

${ }^{43}$ Ibid., p. 236.
} 


\section{Le film de 1942 : Le mariage de Chiffon}

Pour comprendre la représentation des personnages féminins dans le film homonyme de 1942, il faut tenir compte du moment de sa sortie en salle $^{44}$. Nous sommes en pleine guerre mondiale. Les Allemands ont occupé Paris. Il fallait dans ce contexte un film qui puisse entretenir l'audience sans offenser les sensibilités ni éveiller des soupçons, qu'ils fussent pour ou contre les envahisseurs. De ce fait, même l'engagement politique de l'oncle Marc (qui se présente aux élections dans le roman) disparait dans le film, où le héros ne s'occupera que de construire une machine capable de voler ${ }^{45}$. Le déroulement de l'histoire semble avoir avancé dans le temps d'un ou deux lustres. Il en résulte d'autres petits changements dans la reconstitution de l'atmosphère de l'époque : la calèche, la victoria et le landau du roman partagent les chemins avec des automobiles du début de siècle dans le film.

Mais le plus remarquable de ce long métrage est sans doute l'influence de la filmographie américaine de l'époque, qui s'y laisse sentir de façon très frappante. Ainsi, l'ensemble du film est conçu comme une comédie romantique, délassante, gracieuse, égayante, plaisante et sans prétentions de fidélité littéraire. Il n'est pas surprenant alors que l'amabilité et l'élégante légèreté du film soient les traits rehaussés pour décrire cette charmante production ${ }^{46}$, qui ne vise pas la critique sociale, si présente pourtant dans le roman. La caractérisation des personnages apparaîtra, de ce fait, sensiblement simplifiée et stéréotypée, tel que nous le verrons par la suite.

\footnotetext{
${ }^{44}$ Ce long métrage est sorti en salle le 6 août 1942 au cinéma Balzac, à Paris.

${ }^{45}$ Le journal L'Aérophile recueille un article sur le vol du modèle construit pour le film : « Car il faut vous dire que l'aéroplane Henry Farman 1911 reconstitué qui vola le 24 juin 1942 à Vincennes quitta le sol pour les prises de vues du film "Le Mariage de Chiffon" dont on tournait le dernier bout. Le metteur en scène, grâce à une figuration habilement conçue avait parfaitement retracé l'ambiance de l'époque (...). C'est la "Vieille Tigre" Louis Gaubert l'un de nos plus glorieux pilotes, que les réalisateurs du "Mariage de Chiffon" avaient désigné pour chercher et trouver un constructeur capable de reconstituer la machine, malgré les temps difficiles que nous traversons» ( Raymond SALADIN, « Un biplan Henry Farman Modèle 1911 a volé le 24 juin à Vincennes ». L'Aérophile. La revue d'aéronautique la plus ancienne du monde. 50e Année. Juin 1942. p. 166).

${ }^{46}$ Nous signalons, par exemple, le commentaire recueilli dans le Blog Avis sur des films, publié par Christophe le 27/09/2011 dans un article intitulé « Le mariage de Chiffon (Claude Autant-Lara, 1942)» : «Ce qui, outre le dynamisme de la mise en scène, distingue Le mariage de Chiffon des films d'Autant-Lara des années 50 fustigés par Truffaut est l'amabilité de l'ensemble des personnages (à l'exception certes de la mère crispée sur sa position sociale). Même le désir du colonel pour une femme qui pourrait être sa fille n'est pas montré comme sordide. Tout est léger, élégant, guilleret (et sauvé de l'inconséquence par la parfaite rigueur de l'écriture) ». Cf. https:// filmsnonutc.wordpress.com/2011/09/27/la-mariage-de-chiffon-claude-autant-lara-1942/.
} 
Les libertés que le directeur a prises pour verser le roman de Gyp à l'écran surgissent dès les premières scènes du film, entièrement inventées et qui rappellent celles des comédies de Hollywood dans leur naïveté, leur humour innocent et leur charmante candeur : c'est la nuit, il pleut à verse, et la petite Chiffon tombe dans la rue et cherche le soulier qu'elle vient d'égarer dans une flaque d'eau. Le duc d'Aubières qui voit la scène l'aide à chercher la chaussure avec succès. Bien entendu il feindra de ne pas trouver le soulier afin de pouvoir porter la jeune fille dans ses bras jusqu'à la maison. Dans la prétendue obscurité de la nuit, la jeune fille ne peut pas voir le visage de son âgé mais charmant compagnon, qui par la suite la demandera en mariage. L'épisode du soulier égaré permettra d'introduire dès le début du film le personnage de la rivale, Madame de Liron, par le biais d'une autre péripétie totalement inventée. Le duc D'Aubières loge, sans le savoir, dans le même hôtel que le héros, qui passe la nuit avec son amante dans une chambre voisine. La coïncidence veut que les chaussures de Madame de Liron soient identiques à celles de Chiffon. Quand le duc D'Aubières, qui conservait encore le soulier de Chiffon dans sa poche, découvre devant la porte de la chambre voisine le même soulier, il se produit une scène qui vise l'hilarité de la situation : à un moment donné il y aura trois chaussures identiques sur le palier. Ici aussi, la scène reprend l'humour caractéristique des films romantiques américains. Cet épisode, qui n'aura aucun retentissement sur le déroulement de l'action, n'est qu'un clin d'œil du metteur en scène à ces comédies américaines qui faisaient le charme de l'audience de l'époque.

Il s'agit en tout cas aussi d'un procédé qui prouve la volonté de Claude Autant-Lara de revêtir le film d'un humour aimable, façonné sur le modèle américain. D'autres touches d'humour éparpillées tout le long du film dévoilent cette même volonté. Ainsi, le personnage du domestique, Jean ${ }^{47}$, rassemble autour de lui des moments d'un comique tendre et attachant. Mais ce qui est plus important, ce procédé permet d'expliquer aussi la caractérisation stéréotypée des personnages féminins dans le film, tel que nous l'avions annoncé auparavant.

Odette Joyeux est l'actrice choisie pour jouer le rôle de l'héroïne. Il faut signaler, d'emblée, qu'elle est née en 1914, ce qui veut dire qu'elle avait environ 28 ans quand elle a joué le rôle de Chiffon. Ce détail s'avère fondamental pour comprendre les stratégies interprétatives dont elle a fait usage afin d'accorder une apparence de crédibilité à son personnage, âgé

\footnotetext{
${ }^{47}$ Le personnage de Jean est interprété par Pierre Larquey. Dans le film, le domestique joue un rôle plus déterminant dans le déroulement de l'histoire du moment que c'est lui qui dévoile au duc D'Aubières les vrais sentiments de Chiffon.
} 
de 16 ans et demi. Les gestes, le ton, les attitudes de l'actrice visent surtout l'innocence de la jeunesse presque enfantine de la fillette. Le résultat de cet effort pour se rajeunir au moyen du ton et des gestes rehausse surtout la naïveté affectée du caractère représenté : une coquetterie de jeune femme qui dégage une charmante féminité, mais qui reste bien éloignée pourtant des manières spontanées, parfois abruptes, de l'héroïne adolescente du roman, et cela en dépit des réponses impertinentes du personnage qui ont été reprises dans le film, avec plus ou moins d'exactitude. C'est pourquoi il est très difficile de retrouver dans l'interprétation aimable et féminine de cette actrice les traits autobiographiques accordés par Gyp à sa Coryse, comme le sont entre autres son esprit indocile, ses propos critiques, son langage effronté, ses manières quelque peu inconvenantes, ou encore son insouciance pour les conventions sociales.

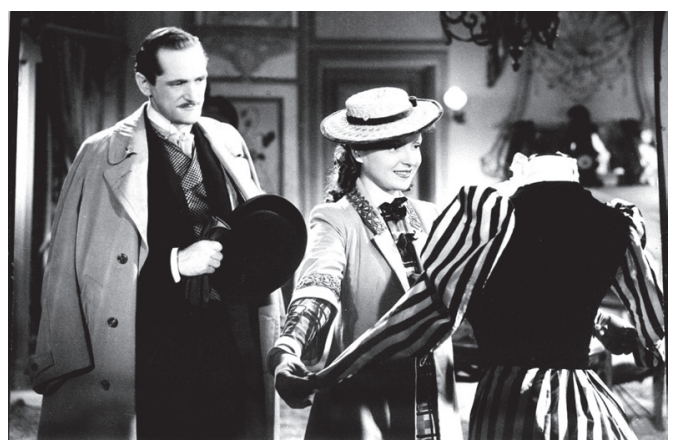

L'oncle Marc (Jacques Dumesnil) et Chiffon $(\text { Odette Joyeux })^{48}$

En outre, les cheveux bruns, soigneusement recueillis dans une queue, frisée de boucles et ornée d'un nœud, n'arrivent pas non plus à reproduire le symbolisme des cheveux blonds en liberté de la Chiffon de Gyp, qui représentaient aussi bien son désir de liberté et sa spontanéité, que son jeune âge. En conséquence, l'interprétation de cette actrice, associée à la mise en scène de Claude Autant-Lara, aboutissent à une représentation de l'héroïne qui demeure beaucoup plus rapprochée des comédies romantiques de l'époque et

\footnotetext{
${ }^{48}$ Page web : http://www.notrecinema.com/communaute/v1_detail_film.php3?lefilm=1234. Photo : http://www.notrecinema.com/images/filmsi/le-mariage-de-chiffon_212325_35636.jpg.
} 
de l'image de la femme du temps de la sortie du film que de la représentation fidèle du personnage romancé.

Cette représentation stéréotypée de l'héroïne atteint aussi le personnage de Madame de Bray, interprété par Suzanne Dantès. L'actrice offre l'image d'une femme âgée, dont le caractère fort et imposant est tout aussi représentatif des modèles équivalents des films américains. Les traits spécifiques d'antagonisme avec Chiffon, présents dans le roman et qui étaient la cible des attaques de Gyp du moment qu'ils renvoyaient à ses expériences personnelles, s'effacent dans le film en faveur d'une caractérisation humoristique, quelque peu histrionique, dépourvue de véritable volonté critique mais tout aussi charmante dans sa représentation stéréotypée. De son côté, Monette Dinay, dans le rôle de Madame de Liron, est caractérisée comme une blonde platine, représentation qui correspond à l'image de la femme sensuelle popularisée dans les films américains par la célèbre Jean Harlow ${ }^{49}$.

C'est ainsi que la représentation de ces trois personnages féminins dans le film ne renvoie pas tant au roman, quant à l'image épocale (celle des années 40) des rôles féminins auxquels elles s'associent, image mentale des spectateurs issue de, et popularisé par la filmographie américaine des années $30-40$.

Dès lors, il se produit une inversion de la représentation des atouts physiques de l'hérö̈ne et de sa rivale: si dans le roman Coryse était une adolescente un peu anguleuse, blonde, plutôt grande et forte, rebelle, critique et contestataire, dans le film elle deviendra une aimable petite brune, féminine et coquette, enfantine dans cette volonté de l'actrice de se rajeunir, mais charmante et plaisante même dans ses caprices ou ses effronteries ; de son côté et à l'inverse, Madame de Liron n'est plus la petite brune rondelette et ambitieuse qui cherchait à devenir le centre d'attention de tous les regards dans le roman, mais une sensuelle blonde platine qui aura une présence beaucoup plus marquée dans le film.

\section{Le téléfilm de 2010 : Le mariage de Chiffon}

Le mariage de Chiffon fut réédité en 1972, ce qui explique l'intérêt renouvelé que ce roman reçut dans les années 70 . Il n'est point surprenant alors que René Lucot, réalisateur de la série Les amours de la Belle Époque,

\footnotetext{
${ }^{49}$ Cette célèbre actrice avait popularisé le rôle de la blonde sensuelle à partir du film de Frank Capra, intitulé justement La blonde platine, de 1931. Jean Harlow deviendra l'icône de la sensualité de l'époque, voluptueuse et féline, qui sous-tend un érotisme latent mais très marqué.
} 
de 1979, lui dédie un des 65 épisodes qui composent cette série télévisée sur Antenne 2. Quelques décennies plus tard, ce même roman sera choisi pour faire partie d'une autre série télévisée : Au siècle de Maupassant. Contes et nouvelles du XIXe siècle. L'épisode du Mariage de Chiffon, tourné en 2009 pour la seconde saison de la collection, fut émis sur France 2 le mercredi 31 mars $2010^{50}$.

Il faut noter d'emblée que cette dernière version du Mariage de Chiffon est beaucoup plus ancrée sur le roman que le film de 1942. Déjà la publicité de la collection fait référence à cette circonstance en insistant sur le choix de «bijoux de la littérature française » pour en offrir des films « exceptionnels ». L'on comprend alors cette volonté de fidélité littéraire et cela malgré les quelques libertés prises, comme celle de l'occupation de l'oncle Marc, qui se consacre ici à sa passion pour la photographie. D'autre part, la présence de personnages comme le père de Ragon prouve aussi cette résolution de reproduire au moins quelques-uns des propos critiques de Gyp.

Ainsi, la première scène du téléfilm renvoie directement à celle du livre : Madame de Bray discute avec son mari, l'oncle Marc et Coryse la proposition du duc d'Aubières, qui veut épouser la jeune fille. Le rôle de l'héroïne retombe cette fois-ci sur la jeune actrice Christa Théret, qui est née en 1991. Elle a, donc, environ 18 ans quand elle a tourné cette série et n'a pas besoin, par conséquent, de feindre une jeunesse qu'elle possède déjà, ce qui tourne au profit de la crédibilité du personnage. En outre, elle est blonde comme la Coryse de Gyp : ses beaux cheveux d'un blond vénitien, longs et souvent ébouriffés, renvoient aussi à la représentation fidèle de la jeune fille de l'histoire. Il en résulte les conditions idéales pour offrir l'image de la figure féminine cherchée par l'auteur dans son ouvrage. Cependant, il n'en sera pas tout à fait le cas. Malgré ces efforts évidents de fidélité littéraire, l'interférence épocale se laisse aussi sentir dans cette dernière version. Nous sommes maintenant au début du XXIe siècle : plus d'un siècle s'est écoulé depuis la parution du roman et, comme il est souvent le cas, les metteurs en scène et les acteurs ne peuvent pas se dégager complètement de la cosmovision de l'époque où ils vivent. C'est ce qui s'est passé avec le film de 1942. Il en sera de même dans ce téléfilm de 2010, bien que à un moindre degré, il est vrai.

\footnotetext{
${ }^{50}$ Alexandre Raveleau commente le succès de cette émission dans un article de Toutlatele. News $T V$ : « La soirée a démarré avec le Mariage de Chiffon. La fiction mise en scène par Jean-Daniel Verhaeghe réunissait Christa Theret et Hippolyte Girardot. L'adaptation du classique de Comtesse de Mirabeau-Martel a réuni 4 millions de téléspectateurs, soit $15.5 \%$ de part de marché ». Cf. http://www.toutelatele.com/le-mariage-de-chiffon-et-un-gentilhomme-bien-discrets-24033.
} 
En fait, dans ce téléfilm Chiffon assure avoir « à peine 18 ans ». De toute évidence, cela découle d'une volonté formelle de la part du directeur de respecter la sensibilité de l'audience et la bienséance de notre société actuelle. Rappelons que de nos jours les cas d'abus d'enfants sont malheureusement de mise. Comme le public du XXIe siècle est très conscient de ce danger, il était important que l'hérö̈ne fût majeure. Une Coryse mineure aurait pu susciter la critique (une accusation de promotion de la pédérastie, par exemple), d'autant plus que le héros de l'histoire a la quarantaine. D'autre part, cette petite liberté ne change rien à l'histoire et permet au demeurant de faire coïncider l'âge du personnage avec celui de l'actrice, ce qui à notre avis est une heureuse idée.

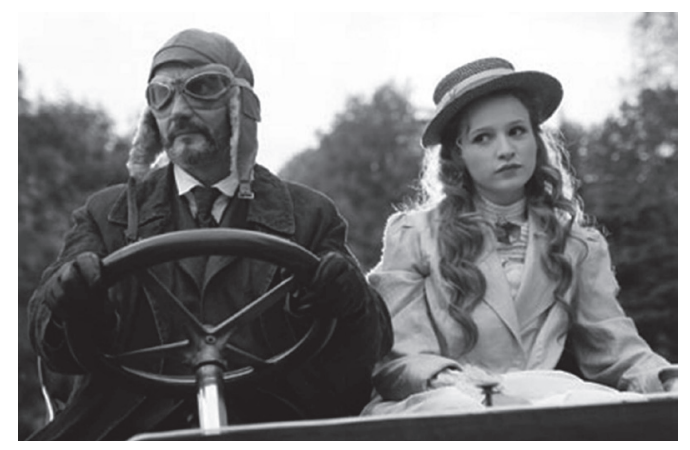

L'oncle Marc (Hippolyte Girardot) et Chiffon (Christa Théret) ${ }^{51}$

Et pourtant, malgré cette coïncidence d'âge, le metteur en scène a trouvé bon de faire souvent tourbillonner et sautiller ${ }^{52}$ la jeune actrice lors de ses interventions. Ses allures ressemblent alors plutôt à celles d'une fillette de 7 ou 8 ans et le résultat est quelque peu artificiel : faire virevolter et voltiger constamment une jeune fille de dix-huit ans nous semble une mesure inutilement forcée ${ }^{53}$, du moment que, tel que nous l'avons signalé, Christa

\footnotetext{
${ }^{51}$ Page web : http://www.toutelatele.com/le-mariage-de-chiffon-et-un-gentilhomme-bien-discrets24033. Photo : http://www.toutelatele.com/IMG/arton24033.jpg

${ }^{52}$ Il n'y a qu'une seule occasion dans le roman où Coryse « esquissa un petit pas guilleret » (138), et c'est celle du moment où elle va annoncer l'héritage de l'oncle Marc aux dames rassemblées à la chapelle des Jésuites. Mais cette conduite n'est que le reflet de la joie anticipée qu'elle éprouve à imaginer l'effet que la nouvelle va produire chez ces dames.

${ }^{53}$ Il se peut que le metteur en scène ait voulu transmettre ce moment évolutif de l'hérö̈ne du roman, où Chiffon est encore en pleine métamorphose : son attitude et ses allures parfois enfantines
} 
Théret a exactement le même âge que son personnage, et que la fraîcheur de son visage aurait suffit amplement à offrir l'image de sa jeunesse, puis qu'elle est réelle, ainsi que celle de la fraîche innocence présupposée à une jeune fille de la fin du XIXe siècle.

Un autre changement, celui qui concerne Madame de Liron, trahit la même volonté d'adapter le roman à la mentalité de notre siècle. Le rôle de la rivale est interprété par Christelle Cornil : elle est grande et d'un blond plus pâle que celui de Coryse, ce qui rappelle la caractérisation du film de 1942. Toutefois, il intéresse de signaler que dans la version de 2010 on lui fournit un prénom, Adèle, qui n'est pas mentionné dans le roman. Mais ce qui est plus frappant c'est qu'elle soit devenue célibataire dans le téléfilm. En conséquence, elle sera adressée comme Mademoiselle de Liron. Ce détail, qui a l'air gratuit et sans importance, permet cependant de mieux transmettre l'intérêt que ce personnage porte à l'oncle Marc. Dès lors, Mademoiselle de Liron ne sera plus seulement sa maîtresse, mais aussi la femme qui le poursuit afin de «l'accrocher ». La rivalité est de ce fait beaucoup plus développée et viscérale dans cette dernière adaptation. Ici, la représentation de ce personnage est celle d'une « vraie » rivale de l'héroïne, du moment que les amantes mariées, n'ayant aucune volonté de divorcer dans le but d'épouser les héros, ne semblent plus qu'un accessoire démodé et un défi bien faible dans la conception amoureuse de notre société actuelle.

Quant à Madame de Bray, il est surprenant de noter à quel point on lui accorde une place privilégiée dans le téléfilm. Le rôle est interprété par Christiane Millet, qui brode sa caractérisation avec des manières affectées et des airs de grandeur. Elle représente ainsi parfaitement l'hypocrisie sociale du personnage et son amour pour les apparences. De nombreuses scènes reprennent avec soin les petits manèges de la marquise et sa complicité avec le Jésuite, rapport qui est encore plus poussé ici que dans le roman ${ }^{54}$. De ce fait, Madame de Bray devient le personnage qui renvoie le mieux à celui de l'ouvrage de Gyp et à son discours critique. Et cependant, en accentuant la connivence échangée entre cette vaine aristocrate provinciale et le père de Ragon — qui partage avec la marquise une place privilégiée dans le téléfilm - cette adaptation ne fait que traduire le goût foncièrement anticlérical du public du XXIe siècle, tout en

évoluent jusqu'à aboutir à celles plus mûres de la jeune fille, déjà femme amoureuse, de la fin de l'histoire.

${ }^{54}$ Une scène du téléfilm — inexistante dans le roman — vise particulièrement à rehausser cette connivence : celle où la marquise partage ses ambitions avec le père de Ragon, qui lui offre son conseil et son appui. Dans un geste de gratitude, Madame de Bray lui donne une enveloppe avec de l'argent. 
conservant en grande mesure la fidélité littéraire ${ }^{55}$. Notons, en revanche, que le film de 1942 n'avait même pas eu l'idée d'introduire le personnage du Jésuite, pourtant très présent dans l'histoire.

\section{Conclusions}

S'il y a une conclusion à tirer de tout ce qui a été dit, c'est sans doute celle du rapport existant entre la cosmovision épocale du récepteur et la représentation des figures féminines du roman. Remarquons que nous entendons ici par récepteur non seulement l'audience, mais aussi et surtout le metteur en scène - ou le directeur - de chacune des deux adaptations à l'écran analysées, du moment que c'est lui qui interprète le texte écrit en vue d'offrir la représentation des différents personnages et des éléments de l'histoire originale. Voilà sans doute la raison pour laquelle l'on a pu constater de si grandes différences entre les interprétations et représentations féminines du film de 1942 et celles du téléfilm de 2010, différences qui s'écartent à leur tour de la version originale, le roman de 1894.

Déjà le choix des épisodes qui font l'objet du tournage trahit le goût épocal de chaque version : le film de 1942 incorpore de nombreuses péripéties qui visent l'humour aimable et naïf des années 40, dans la ligne des comédies américaines de l'époque. D'autre part, l'histoire est réduite à la seule proposition du duc d'Aubières et à la découverte de la part de Chiffon de l'amour qu'elle porte à l'oncle Marc. Les actrices et leurs interprétations renvoient aussi à l'image mentale que les spectateurs des années 40 ont reçue de la filmographie américaine. Le metteur en scène offre ainsi une représentation assez stéréotypée et aimable des personnages féminins selon leur rôle : l'héroïne est une très féminine et coquette petite brune ; la rivale, une blonde platine ; et Madame de Bray, une imposante figure dont l'histrionisme aimable vise plutôt l'effet comique. Il n'y a pas de fidélité littéraire, ni même de volonté critique. Le but est de distraire l'audience de façon délassante et plaisante.

De son côté, le téléfilm de 2010 a beaucoup plus de prétentions littéraires, ce qui explique l'absence d'épisodes inventés à finalité comique et la présence de personnages comme le père de Ragon et le jeune Barfleur, absents dans le film de 1942. Cette même volonté de fidélité littéraire est à l'origine

\footnotetext{
${ }^{55}$ Toutefois, tel que nous l'avons signalé, le discours anticlérical s'avère bien plus marqué dans le téléfilm du moment que le personnage de l'abbé Châtel n'y apparaît pas. Ce dernier était présenté dans le roman comme un modèle de bon et saint prêtre, la critique de Gyp ne visant que les Jésuites.
} 
de la représentation critique de certains personnages, le plus représentatif étant celui de Madame de Bray. Toutefois, cette approche respectueuse de la source textuelle n'est pas accidentelle. Elle découle manifestement du goût de notre époque, qui se plaît à récupérer les ouvrages originaux pour en offrir des adaptions fidèles, avec une reconstruction minutieuse de l'ambiance épocale dans le but de fournir une atmosphère aussi véridique et authentique que possible. C'est sans doute aussi l'extrême sensibilité de notre société à l'égard de comportements criminels contre l'enfance, malheureusement étendus de nos jours, qui pourrait expliquer la décision du directeur d'augmenter l'âge de l'héroïne d'un an et demi, en la faisant majeure, au lieu de mineure. Finalement, cette même cosmovision épocale expliquerait aussi le haut degré de délectation dans l'exploitation d'un discours anticlérical, présent dans le roman mais qui est mis en relief de façon très marquée dans cette dernière version.

De ce fait, les représentations féminines et aussi l'ensemble de ces deux adaptations à l'écran diffèrent à un tel point que l'on croirait avoir affaire à deux romans différents. On ne peut que conclure en soulignant que la représentation des personnages, de l'histoire et du texte se trouve surtout dans le regard transformateur du metteur en scène, qui imprime l'adaptation filmée de la cosmovision, la mentalité, et le goût non pas tant de l'époque du texte romancé, mais aussi et surtout de la sienne.

\section{Références bibliographiques}

CHRISTOPHE, « Le mariage de Chiffon (Claude Autant-Lara, 1942)». Blog : Avis sur des films. Posté le 27/09/2011. Consulté le 04/12/2014. https:// filmsnonutc.wordpress.com/2011/09/27/la-mariage-de-chiffon-claudeautant-lara-1942/.

Lucien CORPECHOT, «Le souvenir de Gyp », dans La revue de Paris, Trenteneuvième année, Tome quatrième, juillet-août 1932, pp. 434-441.

GYP, Le Mariage de Chiffon, Nelson Éditeurs \& Calmann-Lévy Éditeurs, Paris, 1917.

IMDв, « Le mariage de Chiffon (1918). Full Cast and Crew ». Consulté le 4/12/2014. http://www.imdb.com/title/tt0794312/fullcredits?ref_=tt_cl_ sm\#cast.

INA.FR, «Face à vous: Henri Spade ». 13/12/1919. Consulté le 4/12/2014. http://www.ina.fr/video/CPB79056414.

Lucienne MAZENOD, et Ghislaine SCHOELLER, Dictionnaire des femmes célèbres, Éditions Robert Laffont, Paris, 1992. 
NOTRE CINÉMA, « Le mariage de Chiffon ». Consulté le 4/12/2014. http:// www.notrecinema.com/communaute/v1_detail_film.php3?lefilm=1234

Alexandre RAVELEAU, « Le mariage de Chiffon et un Gentilhomme bien discrets ». Toutelatele. News TV. Publié le 1/04/2010. Consulté le 4/12/2014. http://www.toutelatele.com/le-mariage-de-chiffon-et-un-gentilhommebien-discrets-24033.

Raymond SALADIN, « Un biplan Henry Farman Modèle 1911 a volé le 24 juin à Vincennes ». L'Aérophile. La revue d'aéronautique la plus ancienne du monde. 50 Année. Juin 1942, p. 166. 